\title{
Epidemiological Characteristics, Resistance Patterns and Spread of Gram-Negative Bacteria Related to Colonization of Patients in Intensive Care Units
}

\author{
Quésia Souza Damaceno1 ${ }^{*}$, Jacques Nicoli², Adriana Cristina Oliveira1 \\ ${ }^{1}$ Departamento de Enfermagem Básica, Escola de Enfermagem da Universidade Federal de Minas Gerais, \\ Belo Horizonte, Brazil \\ ${ }^{2}$ Departamento de Microbiologia, Universidade Federal de Minas Gerais, Belo Horizonte, Brazil \\ Email: ${ }^{*}$ qdamasceno@yahoo.com.br
}

Received 24 January 2015; accepted 15 February 2015; published 25 February 2015

Copyright (C) 2015 by authors and Scientific Research Publishing Inc.

This work is licensed under the Creative Commons Attribution International License (CC BY).

http://creativecommons.org/licenses/by/4.0/

(c) (;) Open Access

\section{Abstract}

Our aim was to determine the epidemiological characteristics, the resistance patterns and the spread of Gram negative bacteria related to colonization of patients in adult Intensive Care Units. Methods: A prospective cohort of patients colonized and/or infected with Gram negative bacteria was conducted at two adult ICUs from hospitals in Brazil (April 2012 to February 2013). Nasal, groin and perineum swabs were performed. Samples were incubated on MacConkey and cetrimide agar $\left(48 \mathrm{~h}\right.$ at $\left.37^{\circ} \mathrm{C}\right)$ and identification tests (Vitek-BioMérieux), antibiogram (Bauer-Kirby method), Carba NP test, Polymerase Chain Reaction (PCR) and sequencing were performed. The patterns of resistant microorganisms were compared by rep-PCR (Diversilab). Results: There were 53 cases of colonization. In these cases, we identified imipenem-resistant Acinetobacter baumannii (51\%), Pseudomonas aeruginosa (32\%), Klebsiella pneumoniae ESBL (38\%) or imipenem resistant (5.6\%). The use of antimicrobials and medical devices were related to colonization $(p<0.05)$. The resistance patterns expressed by Klebsiella pneumoniae were ESBL (CTX-M, SHV e TEM) and $\mathrm{KPC}_{2}$. A verified profile of Acinetobacter baumannii was related to OXA-23 and OXA-253 (OXA-143 variant). The profiles ESBL and $\mathrm{KPC}_{2}$ expressed by Klebsiella pneumoniae were distributed between the both ICUs. The distribution of OXA-23 and OXA-253 was verified only in one ICU. The similarity of strains ranged from $\mathbf{8 0} \%$ to $95 \%$, highlighting the horizontal transference of these microorganisms.

\section{Keywords}

Intensive Care Unit, Bacterial, Drug-Resistance, Gram-Negative Aerobic Bacteria, Colonization

\footnotetext{
${ }^{*}$ Corresponding author.
}

How to cite this paper: Damaceno, Q.S., Nicoli, J. and Oliveira, A.C. (2015) Epidemiological Characteristics, Resistance Patterns and Spread of Gram-Negative Bacteria Related to Colonization of Patients in Intensive Care Units. Advances in Infectious Diseases, 5, 14-20. http://dx.doi.org/10.4236/aid.2015.51002 


\section{Introduction}

Among the Gram-negative bacteria the Enterobacteriaceae (Escherichia coli, Klebsiella spp., Enterobacter spp., Proteus spp., Serratia spp.) and non-fermenters (Pseudomonas aeruginosa and Acinetobacter baumannii) are frequently associated with infections in immunocompromised patients, especially in Intensive Care Units (ICU) and, in a several cases show multidrug-resistance profile [1].

The multidrug-resistance among species of Enterobacteriaceae has become a global challenge, with high levels of resistance against carbapenems worldwide. The resistance against carbapenems may be facilitated due the widespread use in treatment of infections caused by Enterobacteriaceae or non-fermenters (A. baumannii and $P$. aeruginosa) with a large profile of resistance to $\beta$ lactams [2].

Similarly, the emergence and spreading of carbapenemases among Gram-negative bacteria can be verified, especially with Enterobacteriaceae species. The first report of carbapenemase was registered with Klebsiella pneumoniae in 2001 in the USA. The KPC-2 is the most frequent variant among class A carbapenemases. The genes blaKPC can be located in a wide variety of plasmids, facilitating further resistance [3] [4].

Although the first report of carbapenemases was registered with Enterobacteriaceae, the others Gram-negative bacteria, such as $A$. baumannii and P. aeruginosa, can also produce this enzyme. The class D carbapenemases or Oxalinases (OXAs) are frequently found in both Gram-negative bacteria. The OXAs have a large spectra of antibiotic hydrolyze, including carbaphenems. Currently, more than 150 OXAs variants have already been described [4] [5].

According to the results of the Program of Epidemiological Surveillance and Bacterial Drug Resistance for Latin America and Brazil, the non-fermenter multidrug-resistant Gram-negative Bacilli and Enterobacteriaceae with levels of extended-spectrum beta-lactamase (ESBL) constitute the main problem of resistance to antimicrobials in these countries [6] [7].

The identification and characterization of Gram-negative microorganisms with resistance patterns to antimicrobials may improve the management of sources and spreading, enabling plans infection control measures plans in settings with important prevalence of these microorganisms. Therefore, it was aimed to determine the epidemiological characteristics, the resistance patterns and spread of Gram negative bacteria related to colonization of patients in adults Intensive Care Unit.

\section{Materials and Methods}

A prospective cohort study was approved by the Ethics Committee (0386.0.203.000-11) and performed in two adults Intensive Care Units in two hospitals in Belo Horizonte, Brazil (April 2012 to February 2013). Patients or their next of kin were requested to give consent prior to sampling. All consenting patients colonized or infected with Gram-negative microorganisms with resistance patterns and who were more than 18 years old were followed during their length of stay in the ICU or in wards where the patients was transferred after being discharged from the ICU.

Patient characterisation data were collected via chart review, including age, sex, monitoring by medical devices (mechanical ventilation, venous catheter, drains and, nasal and indwelling catheter), severity index (measured according to the classification of the Average Severity Index Score-ASIS), antimicrobial use (class, previous use, use during the length of stay in the ICU and time of use), and the occurrence of infection by MDROs. The results concerning occurrence of infection were retrieved from patient charts and by checking hospital surveillance data.

\subsection{Sampling and Microbiological Testing}

The patients were evaluated with bi-weekly microbiological cultures after confirming their colonization status from identifying imipenem-resistant $K$. pneumoniae or ESBL, imipenem-resistant $P$. aeruginosa and A. baumannii.

One swab (Haste Plástico-J. P) was rotated two revolutions in the nasal membrane, another was used for the groin and a third was rotated from the anterior to posterior perineum. The samples were plated in the following dilutions: nasal and groins $\left(10^{2}\right.$ and $\left.10^{4}\right)$ and perineum $\left(10^{4}\right.$ and $\left.10^{6}\right)$. Each dilution was distributed with100 $\mu$ l on plates: MacConkey agar (K. pneumoniae and A. baumannii), cetrimide ( $P$. aeruginosa), incubated at $37^{\circ} \mathrm{C}$ for $48 \mathrm{~h}$. Each selective medium and dilutions were previously standardized using cultivated duplicate plates $K$. 
pneumoniae, A. baumannii and $P$. aeruginosa. Identification was performed using Gram stain, and biochemistry cards (Vitek ${ }^{\circledR} 2$ for Gram negative-bioMérieux, Marcy L’Etoile, France).

After each bacterium was identified, the Bauer-Kirby antibiogram method was used (imipenem/meropenem and ceftazidime for $P$. aeruginosa, K. pneumoniae and A. baumannii), third generation cephalosporin-ceftazidime, cefotaxime and ceftriaxone for K. pneumoniae) [8]. The Carba NP test was performed to verify imipenem hydrolysis [9].

Polimerase Chain Reaction (PCR): All samples identified as K. pneumoniae were screened with the following genes: NDM, IMP, VIM, KPC and OXA 48 e for those identified as Acinetobacter baumannii were screened with the genes OXA-23, OXA-40, OXA-58 and OXA-143, as previously described by Odeh et al., 2002 [10].

\subsection{Samples Sequencing}

Positive samples for the following genes (NDM, IMP, VIM, KPC, OXA-23, OXA-40, OXA-48, OXA-58 or OXA-143) were sequenced using their respective primers (forward and reverse) as previously described by Odeh et al., 2002 [10].

\subsection{Strains Similarity}

The Diversilab (Biomériaux) technique was used to analyze strain similarity. The Diversilab is a technique based on the principles of the repetitive sequence based PCR (rep-PCR), that amplifies different fragments sizes of non coded DNA (specific for each specie), offering a quick distinction among species and subspecies (Biomériaux, 2013).

\subsection{Statistical Analysis}

Statistical analysis was performed using the $\mathrm{T}$ student test, $\mathrm{X}^{2}$ test or Fisher exact test. The predictive factors related to colonization or infection with MDROs were analyzed by logistic regression. Bivariate analysis was used to identify the variables with $\mathrm{p}<0.25$ for insertion into a multivariate model. The significance level was $5 \%$ to $95 \%$ confidence intervals. Analyses were performed using STATA (11.0).

\section{Results}

Among 53 evaluated patients 51.0\% (27/53) showed colonization with A. baumannii and 32.0\% (17/53) by $P$. aeruginosa, both carbapenems resistant and 38.0\% (20/53) had colonization with K. pneumoniae ESBL or KPC $5.6 \%(3 / 53)$.

The main characteristics of the evaluated patients $(n=53)$ were: predominance of male gender $(52.0 \%)$, at time of ICU admission $64.0 \%$ of patients were from another sector in the hospital and, for $72.0 \%$ of patients the previous use of antimicrobials in hospital sectors prior to ICU transferences was not verified. During the length of stay in the ICU, we verified an important use of meropenem $(p<0.05)$ among others antimicrobials, with a mean of 24 doses per patient.

A total of 114 samples were related to Gram negative bacilli with resistance profile (resistance to third generation cephalosporin and carbapenem). Among these samples 80 we identified as third generation cephalosporin and/or carbapenem resistant K. pneumoniae $(\mathrm{n}=41)$ and carbapenem resistant A. baumannii $(\mathrm{n}=39)$.

Five strains of $K$. pneumoniae and six strains of $A$. baumannii were identified in the imipenem hydrolysis. The positive hydrolyses was verified visually by the change of the medium color from red to yellow or orange. The amplification of the DNA of these strains showed presence of genes for antimicrobial resistance such as KPC2 for 3 strains of $K$. pneumoniae. After the sequencing these strains were identified as:

1) Carbapenemase class A-KPC-2 [K. pneumoniae]-Identification in genbank: gb|ACO82075.1|; 2) Betalactamase KPC-2, partial [K. pneumoniae]_Identification in genbank: gb|AFN82205.1| e, 3) Carbapenemase class A-KPC-2 [K. pneumoniae]_Identification in genbank: gb|ACO82075.1|.

Another two strains of the K. pneumoniae PCR results tested positive for the following genes: OXA I, CTXM, SHV e TEM (Table 1).

Among the multidrug-resistant A. baumannii we verified the genes for OXA 23 and OXA 143 (Table 2).

After sequencing, the strain of A. baumanni-Ab 25 was identified as OXA-253; one variant of OXA-143 [A. baumannii] as described by Girlich et al., 2014 [11]. 
Table 1. Expression of genes of resistance to antimicrobials by K. pneumoniae related to colonization of patients in Intensive Care Units-Belo Horizonte, 2014.

\begin{tabular}{ccccccc}
\hline Strains & Oxa1 & Oxa9 & CTXM & SHV & TEM \\
\hline Kp30 & + & - & + & + & + \\
Kp37 & - & - & - & + & + \\
\hline
\end{tabular}

Table 2. Expression of genes of resistance to antimicrobials by A. baumannii related to colonization of patients in Intensive Care Units-Belo Horizonte, 2014.

\begin{tabular}{ccc}
\hline Strains & Oxa 23 & OXA 143 \\
\hline A. baumannii-Ab2 & + & - \\
A. baumannii-Ab25 & + & - \\
A. baumannii-Ab36 & + & + \\
A. baumannii-Ab38 & + & - \\
A. baumannii-Ab39 & + & + \\
\hline
\end{tabular}

Similarly, analyses for multidrug-resistant K. pneumoniae were verified in 3 different patterns. The cross transmission was registered in ICU II with a percentual of similarity higher than 90.0\%, between Kp7 and Kp19 (profile $\mathrm{KPC}_{2}$ ) isolated from two different patients. Another important similarity higher than $80.0 \%$ was verified between Kp4 (isolated from patient colonization in ICU II) and Kp30 (isolated in colonization of another patient in ICU I), suggesting the spread of similar patterns between these ICUs that are located in the same region of Belo Horizonte.

The identified patterns for $A$. baumannii showed a similarity profile of $95.0 \%$. These samples were isolated from colonization cases in different patients in ICU II during the follow up.

\section{Characteristics Related to Patient Colonization in the Intensive Care Unit}

The main characteristics related to colonization of resistant Gram-negative bacilli were the use of medical devices and antimicrobials during the length of stay in the ICUs ( $<<0.05)$. In the colonization with imipenem-resistant $P$. aeruginosa, the presence of pressure ulcers and severity index was highlighted according to the results of bivariate analyses (Table 3).

With imipenem-resistant $P$. aeruginosa, some variables were highlighted in the final logistic regression model, such as the use of mechanical ventilation and fluoroquinolones, which accounted for approximately $32 \%$ of colonization with this microorganism. The main colonization sites by carbapenem resistant $P$. aeruginosa were nares $(\rho: 0.8 ; \mathrm{p}=0.001)$ and groins $(\rho: 0.7 ; \mathrm{p}=0.001)$.

Among colonized patients with imipenem-resistant $K$. pneumoniae or $K$. pneumoniae producing ExtendedSpectrum Beta lactamases, the use of fluoroquinolone during the length of stay in the ICU was the most important characteristic. The groin was the main anatomic site colonized by ESBL or carbapenem resistant K. pneumoniae $(\rho: 0.7 ; \mathrm{p}=0.0001)$.

The presence of pressure ulcers and carbapenems used during the length of stay in the ICUs were also important characteristics of patient colonization with imipenem-resistant $A$. baumannii. In regards to patients colonized with carbapenem resistant $A$. baumannii, the main site for identification of this microorganism were nares $(\rho: 0.7 ; \mathrm{p}=0.001)$ and groins $(\rho: 0.6 ; \mathrm{p}=0.001)$.

\section{Discussion}

In colonization with multidrug-resistant Gram negative bacilli, we highlighted $K$. pneumoniae, with a variety of profiles, such as ESBL (CTXM, SHV e TEM) and $\mathrm{KPC}_{2}$, imipenem resistant $P$. aeruginosa and carbapenem resistant A. baumannii with expressions of the genes OXA 23 and OXA 253 (a variant of OXA-143), distributed 
Table 3. Variables in colonization with Pseudomonas aeruginosa $(\mathrm{n}=17)$, Klebsiella pneumoniae $(\mathrm{n}=23)$ and Acinetobacter baumannii $(\mathrm{n}=27)$-Belo Horizonte, 2014.

\begin{tabular}{|c|c|c|c|c|c|c|}
\hline \multirow{2}{*}{ Variables } & \multicolumn{2}{|c|}{ P. aeruginosa } & \multicolumn{2}{|c|}{ K. pneumoniae } & \multicolumn{2}{|c|}{ A. baumannii } \\
\hline & $\mathbf{N}$ & $\mathbf{p}$ & $\mathbf{n}$ & $\mathbf{P}$ & n & $\mathbf{p}$ \\
\hline Age & 51 & 0.2 & 48 & 0.50 & 61 & 0.30 \\
\hline \multicolumn{7}{|l|}{ Sex } \\
\hline Male & 13 & & 13 & & 16 & \\
\hline Female & 4 & 0.04 & 10 & 0.90 & 11 & 0.60 \\
\hline Length of stay (ICU) & 33 & 0.10 & 23 & 0.40 & 24 & 0.40 \\
\hline Pressure ulcer & 10 & $<0.01$ & 9 & 0.30 & 12 & 0.04 \\
\hline \multicolumn{7}{|l|}{ Medical devices (\% use) } \\
\hline Venous catheter & 13 & 0.04 & 15 & 0.20 & 17 & 0.30 \\
\hline Nasal probe & 14 & 0.01 & 15 & 0.30 & 18 & 0.20 \\
\hline Indwelling catheter & 14 & 0.05 & 12 & 0.10 & 18 & 0.60 \\
\hline Mechanical ventilation & 14 & 0.01 & 13 & 0.80 & 18 & 0.09 \\
\hline Drains & 7 & 0.40 & 60 & 1.0 & 11 & 0.30 \\
\hline Antimicrobials use (days) & 9 & 0.07 & 9 & 0.40 & 10 & 0.20 \\
\hline \multicolumn{7}{|l|}{ Antimicrobials } \\
\hline Penicillin & 9 & 0.30 & 12 & 0.20 & & 0.30 \\
\hline Glycopeptide & - & - & - & - & 0 & - \\
\hline Fluoroquinolone & 11 & $<\mathbf{0 . 0 0}$ & 11 & 0.01 & & 0.60 \\
\hline Carbapenem & 12 & 0.01 & 14 & 0.08 & & $<0.01$ \\
\hline \multicolumn{7}{|l|}{ Severity } \\
\hline $\mathrm{C}$ & 11 & & 19 & & 24 & \\
\hline $\mathrm{D}$ & 5 & & 3 & & 3 & \\
\hline E & 1 & 0.05 & 1 & 0.50 & & - \\
\hline
\end{tabular}

in colonization cases in both ICUs. This profile is similar to those registered throughout Latin America and Brazil [6].

In regards to K. pneumoniae producing carbapenemases, in the last 10 years this profile has been associated with infections with limited therapeutic options, showing multiple mechanisms of resistance [12] [13]. Infections caused by A. baumannii, are associated with mechanical ventilation use, presence of wounds or burns and mainly in critical patients [14] [15].

Among the multidrug-resistant Gram negative bacilli, the ESBL profile was highlighted, such as the CTX-M that have been noted as the most frequent pattern worldwide. The ESBL expression by Enterobacteriaceae is frequent in hospital settings. These enzymes hydrolysis cephalosporins of amplified spectra, such as cefotaxime, ceftazidime and Aztreonam [16]. The expression of CTX-M has been verified since the 1990s, mainly in samples of Escherichia coli [17] [18].

The large expression of ESBL among Gram-negative bacilli increases the carbapenems use, one such antimicrobial options for treatment of infections caused by these microorganisms. The increased use of carbapenems enables the expression of carbapenemases due to selective pressure [17] [18]. In the present follow up the meropenem, one such example of carbapenem was the most used antimicrobial in ICU I and ICU II.

The strain of A. baumannii identified as OXA-253; a variant of OXA-143 [A. baumannii]. Others variants of OXA-143 have been registered in Brazil (OXA-231, in Parana state), Honduras and the United States [19] [20]. The OXA-23 expression is registered all over South America and was the main profile identified in ICU I and ICU II in this follow up [21] [22].

A large variation was found with patterns of multidrug-resistant $K$. pneumoniae. For this microorganism we registered 3 different patterns when we evaluated similarity among the strains. The percentual of similarity ranged from $80.0 \%$ to $90.0 \%$. The highest similarity was verified with strains of $\mathrm{KPC}_{2}$ from two patients in ICU II, suggesting cross transmission of the microorganism. 
The patterns identified for A. baumannii showed high similarity (95.0\%), perhaps the most important patterns related to antimicrobial resistance in A. baumannii and cross transmission in these hospital settings.

Some factors associated with patient conditions in the ICUs may be related to the acquisition of resistant microorganisms, as verified in this follow up; the antimicrobials and medical devices used were important factors in colonization with BGN.

In one evaluation of clinical samples isolated in patients from 18 hospitals in Germany, 54.3\% were identified as ESBL. It was observed that the Gram-negative bacilli carriers had a high length of stay (mean: 37.2 days). The prolonged length of stay in health institutions and the antimicrobials used may increase the risk of multidrug-resistant microorganisms acquisition [23] [24].

In analyzing patients colonization we evaluated the nares, the groins and perineum regarding the low temporal variability of microorganisms in these skin sites. The main sites for identification of BGN, in this study, were the nares and the groins. Therefore, the identification of resistant bacteria in these sites may imply colonization establishment [25] [26].

For further evaluations of patterns and spread of Gram-negative bacilli, it is important to have a longer period of follow up and to perform multicentre studies. Although, in this study we performed a short follow up of patient colonization with multidrug-resistant Gram-negative bacilli, it was possible to verify different patterns of resistance to antimicrobials and its spread throughout two ICUs in Belo Horizonte, Brazil. The identification of multidrug-resistant microorganisms carriers may facilitate the management of infection control measures and reduce the spread of microorganisms and infections.

\section{Acknowledgements}

INSERM 914 “Résistances Emergentes aux Antibiotiques”, Centre National de Référence Associé Résistance aux Antibiotiques, Hopital de Bicetre, Paris, France.

\section{Funding}

Study supported by the Conselho Nacional de Desenvolvimento Científico e Tecnológico (CNPQ)-No. 472823/ 2011-6.

\section{References}

[1] Livermore, D. (2012) Current Epidemiology and Growing Resistance of Gram-Negative Pathogens. Korean Journal of Internal Medicine, 27, 128-142. http://dx.doi.org/10.3904/kjim.2012.27.2.128

[2] Lowe, F.C., Mcgeer, A., Muller, M.P. and Katz, K. (2012) For the Toronto ESBL Working Group. Decreased Susceptibility to Noncarbapenem Antimicrobials in Extended-Spectrum- $\beta$-Lactamase-Producing Escherichia coli and Klebsiella pneumoniae Isolates in Toronto, Canada. Antimicrobial Agents and Chemotherapy, 56, 3977-3980. http://dx.doi.org/10.1128/AAC.00260-12

[3] Yigit, H., Queenan, A.M., Anderson, G.J., Domenech-Sanchez, A., Biddle, J.W., Steward, C.D., et al. (2001) Novel Carbapenem-Hydrolyzing $\beta$-Lactamase KPC-1 from a Carbapenem-Resistant Strain of Klebsiella pneumoniae. Antimicrobial Agents and Chemotherapy, 45, 1151-1161. http://dx.doi.org/10.1128/AAC.45.4.1151-1161.2001

[4] Naas, T., Cuzon, G., Truong, H.V. and Nordmann, P. (2012) Role of ISKpn7 and Deletions in blaKPC Gene Expression. Antimicrobial Agents and Chemotherapy, 56, 4753-4759. http://dx.doi.org/10.1128/AAC.00334-12

[5] Poirel, L., Naas, T. and Nordmann, P. (2010) Diversity, Epidemiology, and Genetics of Class D $\beta$-Lactamases. Antimicrobial Agents and Chemotherapy, 54, 24-38. http://dx.doi.org/10.1128/AAC.01512-08

[6] Sader, H.S., Jones, R.N., Gales, A.C., Silva, J.B. and Pignatari, A.C., Participantes do grupo Sentry (América Latina) (2004) SENTRY Antimicrobial Surveillance Program Report: Latinamerican and Brazilian Results for 1997 through 2001. Brazilian Journal of Infectious Diseases, 8, 25-79. http://dx.doi.org/10.1590/S1413-86702004000100004

[7] Rice, L.B. (2008) Federal Funding for the Study of Antimicrobial Resistance in Nosocomial Pathogens: No ESKAPE. Journal of Infectious Diseases, 197, 1079-1081. http://dx.doi.org/10.1086/533452

[8] Clinical and Laboratory Standards Institute (CLSI) (2011) Performance Standards for Antimicrobial Disk Susceptibility Tests; Approved Standard-Tenth Edition. CLSI Document M02-A10. Clinical and Laboratory Standards Institute, Wayne.

[9] Nordmann, P., Poirel, L. and Dortet, L. (2012) Rapid Detection of Carbapenemase Producing Enterobacteriaceae, Emerging Infectious Diseases, 8,1503-1507. http://dx.doi.org/10.3201/eid1809.120355 
[10] Odeh, R., Kelkar, S., Hujer, A.M., Bonomo, R.A., Schreckenberger, P.C. and Quinn, J.P. (2002) Broad Resistance Due to Plasmid-Mediated AmpC b-Lactamases in Clinical Isolates of Escherichia coli. Clinical Infectious Diseases, 35, 140-145. http://dx.doi.org/10.1086/340742

[11] Girlich, D., Damaceno, Q.S., Oliveira, A.C. and Nordmann, P. (2014) OXA-253, a Variant of the Carbapenem-Hydrolyzing Class D $\beta$-Lactamase OXA-143 in Acinetobacter baumannii. Antimicrobial Agents and Chemotherapy, 58, 2976-2978. http://dx.doi.org/10.1128/AAC.02640-13

[12] Papadimitriou-Olivgeris, M., Marangos, M., Fligou, F., Christofidou, M., Bartzavali, C., Anastassiou, E.D., et al. (2012) Risk Factors for KPC-Producing Klebsiella pneumoniae Enteric Colonization upon ICU Admission. Journal of Antimicrobial Chemotherapy, 67, 2976-2981. http://dx.doi.org/10.1093/jac/dks316

[13] Feldman, N., Adler, A., Molshatzki, N., Navon-Venezia, S., Khabra, E., Cohen, D. and Carmeli, Y. (2013) Gastrointestinal Colonization by KPC-Producing Klebsiella pneumoniae Following Hospital Discharge: Duration of Carriage and Risk Factors for Persistent Carriage. Clinical Microbiology and Infection, 19, E190-E196. http://dx.doi.org/10.1111/1469-0691.12099

[14] Towner, K.J. (2009) Acinetobacter: An Old Friend, but a New Enemy. Journal of Hospital Infection, 73, 355-363. http://dx.doi.org/10.1016/j.jhin.2009.03.032

[15] Kempf, M. and Rolain, J.M. (2012) Emergence of Resistance to Carbapenems in Acinetobacter baumannii in Europe: Clinical Impact and Therapeutic Options. International Journal of Antimicrobial Agents, 39, 105-114. http://dx.doi.org/10.1016/j.ijantimicag.2011.10.004

[16] Paterson, D.L. (2008) The Molecular Epidemiology of Extended-Spectrum Beta-Lactamase Producing Organisms. Enfermedades Infecciosas y Microbiología Clínica, 26, 403. http://dx.doi.org/10.1157/13125635

[17] Lartigue, M.F., Zinsius, C., Wenger, A., Bille, J., Poirel, L. and Nordmann, P. (2007) Extended-Spectrum $\beta$-Lactamases of the CTX-M Type Now in Switzerland. Antimicrobial Agents and Chemotherapy, 51, 2855-2860. http://dx.doi.org/10.1128/AAC.01614-06

[18] Ruppé, E., Pitsch, A., Tubach, F., Lastours, V., Chau, F., Pasquet, B., et al. (2012) Clinical Predictive Values of Extended-Spectrum Beta-Lactamase Carriage in Patients Admitted to Medical Wards. European Journal of Clinical Microbiology \& Infectious Diseases, 31, 319-325. http://dx.doi.org/10.1007/s10096-011-1313-z

[19] Gionco, B., Pelayo, J.S., Venancio, E.J., Caio, R., Gales, A.C. and Carrara-Marroni, F.E. (2012) Detection of OXA-

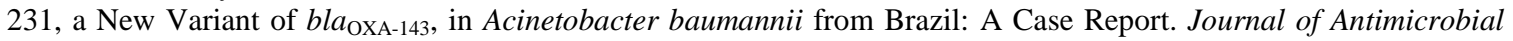
Chemotherapy, 67, 2531-2532. http://dx.doi.org/10.1093/jac/dks223

[20] Zander, E., Bonnin, R.A., Seifert, H. and Higgins, P.G. (2014) Characterization of bla $a_{\mathrm{OXA}-143}$ Variants in Acinetobacter baumannii and Acinetobacter pittii. Antimicrobial Agents and Chemotherapy, 58, 2704-2708. http://dx.doi.org/10.1128/AAC.02618-13

[21] Higgins, P.G., Poirel, L., Lehmann, M., Nordmann, P. and Seifert, H. (2009) OXA-143, a Novel Carbapenem-Hidrolyzing Class D $\beta$-Lactamase in Acinetobacter baumannii. Antimicrobial Agents and Chemotherapy, 53, 5035-5038. http://dx.doi.org/10.1128/AAC.00856-09

[22] Opazo, A., Domínguez, M., Bello, H., Amyes, S.G. and Rocha, G. (2012) OXA-Type Carbapenemases in Acinetobacter baumannii in South America. Journal of Infection in Developing Countries, 6, 311-316.

[23] Sostarich, A.M., Zolldann, D., Haefner, H., Luetticken, R., Schulze-Roebecke, R. and Lemmen, S.W. (2008) Impact of Multiresistance of Gram-Negative Bacteria in Bloodstream Infection on Mortality Rates and Length of Stay. Infection, 36, 31-35. http://dx.doi.org/10.1007/s15010-007-6316-4

[24] Willwmsen, I., Elberts, S., Verhulst, C., Rijnsburger, M., Filius, M., Savelkoul, P., et al. (2011) Highly Resistant Gram-Negative Microorganisms: Incidence Density and Occurrence of Nosocomial Transmission (TRIANGLE Study). Infection Control and Hospital Epidemiology, 32, 333-341. http://dx.doi.org/10.1086/658941

[25] Grice, E.A., Kong, H.H., Conlan, S., Deming, C.B., Davis, J., Young, A.C., et al. (2009) Topographical and Temporal Diversity of the Human Skin Microbiome. Science, 324, 1190-1192. http://dx.doi.org/10.1126/science.1171700

[26] Chen, Y.E. and Tsao, H. (2013) The Skin Microbiome: Current Perspectives and Future Challenges. Journal of the American Academy of Dermatology, 69, 143-155. http://dx.doi.org/10.1016/j.jaad.2013.01.016 
Scientific Research Publishing (SCIRP) is one of the largest Open Access journal publishers. It is currently publishing more than 200 open access, online, peer-reviewed journals covering a wide range of academic disciplines. SCIRP serves the worldwide academic communities and contributes to the progress and application of science with its publication.

Other selected journals from SCIRP are listed as below. Submit your manuscript to us via either submit@scirp.org or Online Submission Portal.
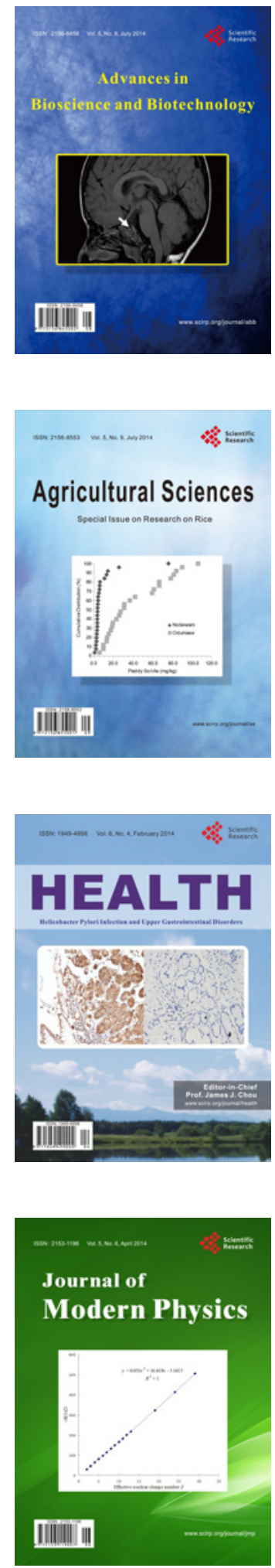


Creative Education
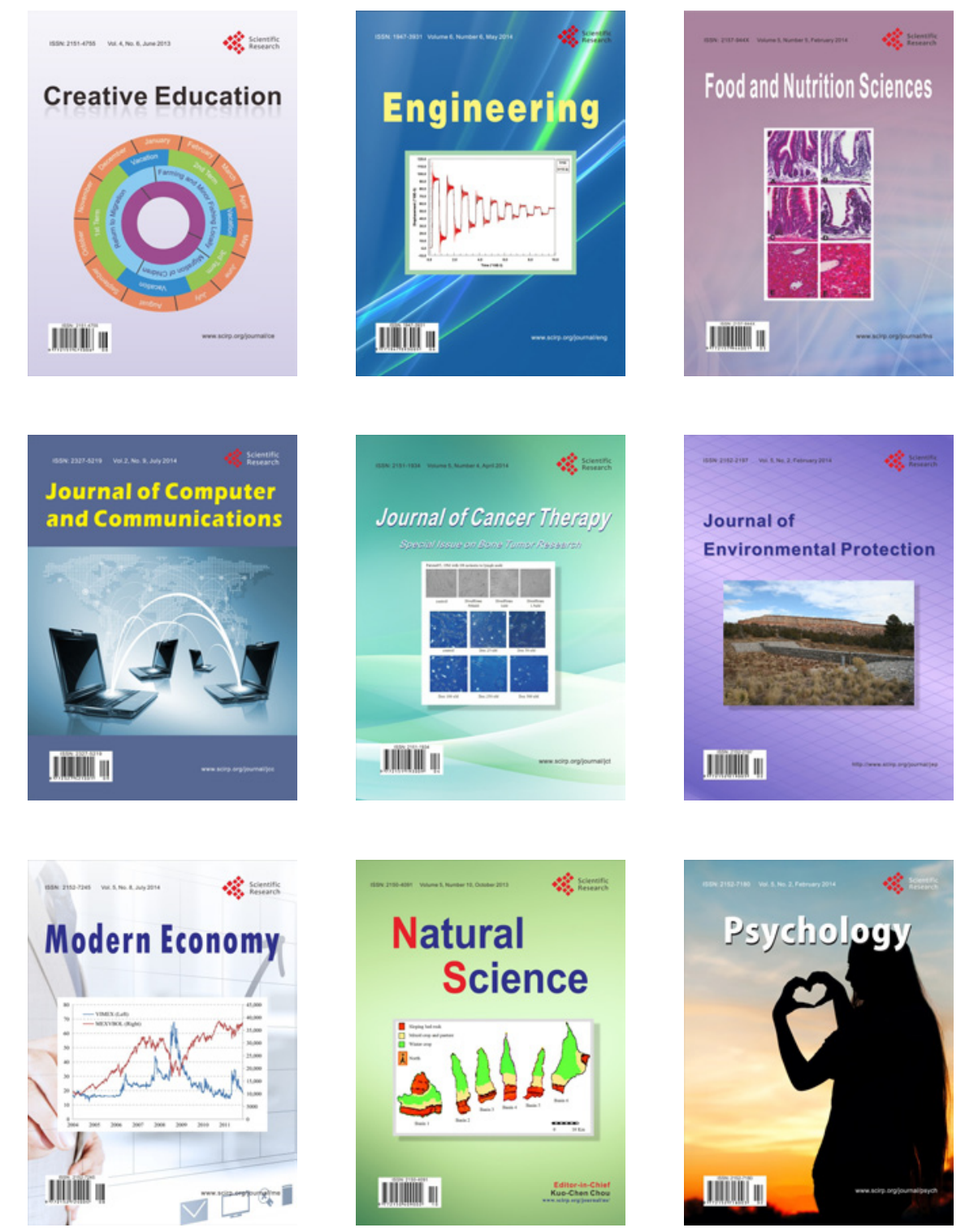